\title{
(5) Metabolic and Kinetic Study of Platelets in Thrombogenesis
}

\author{
Atsushi Kuramoto, M D \\ Department of Medicine, Research Institute for Nuclear Medicine \\ and Biology, Hiroshima University
}

Platelets, the third components of blood cells, are important in the plug formation in hemostasis. Recent advance in this field revealed that these particles derived from the cytoplasma of megakaryocytes in the bone marrow, posess all cell components; mitochondria, ribosome, granules, vesicle systems, glycogen microtubulus, except nucleus. These small cells are active in metabolism to maintain their shape and functions; active transport of bioactive substances, pseudopod formation, release reaction, adhesion, aggregation, retraction, and phagocytosis. Their main energy sources are glucose in plasma and glycogen in the cells. They are active in de novo synthesis of glycogen, protein, and fatty acid.

In the process of thrombogenesis, adhesion of platelet to collagen or other subendothelial tissues, or aggregation of primary step, induce release reaction of mainly nucleotides: ADP, which induces further aggregation of secondary. We presented the results that these primary important step was deeply energy dependent and closely related to the oxidative phosphorylation in the mitochondria via citric acid cycle (CAG). To elucidate the metabolic and kinetic basis of throm. bogenesis it is important to predict episodes and establish the early prophylactic therapy in thrombogenic diseases, which are increaseing in number; cardiovascular and cerebral thrombosis and implantation of artificial thrombogenic organs. We conducted in vitro experiment of glucose metabolism of human platelets and in vivo study of ${ }^{51} \mathrm{Cr}$ labeled platelet survival on the patients with thrombogenic diseases. The platelets were obtained from healthy volunteer and the patients with marked thrombocytotic disorders; chronic myelogenous leukemia (CML), myelofibrosis with myloid metaplasia (MMM) and primary thrombocythemia and vascular and heart valvular diseases. Glucose metabolism was studied by measuring the radioactivity of produced ${ }^{14} \mathrm{CO}_{2}$ and ${ }^{14} \mathrm{C}$-lactate from ${ }^{14} \mathrm{C}$-glucose, with scintillation counter. Lactate and glycogen were determined as was described (Ser. Haemat 4:98, 1971). ADP, collagen, epinephrine, ristocetin and Latex particles of $0.234 \mu$ in size were used as aggregating agents. Kinetic study was performed using ${ }^{51} \mathrm{Cr}\left(\mathrm{Na}_{2} \mathrm{Cr} \mathrm{O}_{4}\right)$ to label separated platelets from ABO compatible donors or autologous blood of 200 to $450 \mathrm{ml}$ in volume. Procedures were as described previously (Acta Haemat Jap 29:664, 1966). Body surface scanning were also done to detect organ distribution of tagged platelets. In vitro study of platelet function of aggregation and adhesion was done according to Born's method and modified Salzmann's method.

\section{RESULTS}

To study the energy metabolism in aggregating platelets, we used differently labeled glucose as the substrate. In platelets, aerobic and anaerobic glycolysis are operating. In the event of aggregation with release reaction increased glucose consumption took place 
especially in CAC. $\mathrm{CO}_{2}$ production by nonaggregating platelets were 26.7 nmoles from ${ }^{14} \mathrm{C}-1$-glucose, $2-3$ nmoles from ${ }^{14} \mathrm{C}$ 2 -glucose, $1.5-1.9$ nmoles from ${ }^{14} \mathrm{C}-3.4$-glucose and $1.5-1.7 \mathrm{nmoles}$ from ${ }^{14} \mathrm{C}$-6-glucose and $1.21-1.55 \mu$ moles lactate per $1 \times 10^{9}$ platelets for 2 hours. ADP, thrombin, collagen and Latex particles did stimulate glucose consumption of ${ }^{14} \mathrm{C}$-6-glucose indicating product increment of twice by collagen and epinephrine and 8 to 10 times increase by Latex particles. No net increase of hexose monophosphate shunt (HMS) was observed by these agents. In contrast, methylene blue stimulated HMS around 10 times, but not of citric acid cycle.

These responses to metabolic stimulators, were inhibited by metabolic inhibitors such as deoxyglucose, iodoacetate, KGN, Amytal $1 \mathrm{mM}$ respectively and prostaglandin $\mathrm{E}_{1}\left(10^{-4}-10^{-6} \mathrm{M}\right)$. Latex particles are the most potent stimulators via phagocytic stimulation of lysosomes (BBA 201:471, 1970). In contrast to phagocytic neutrophils, platelets phagocytosis accompanied citric acid cycle stimulation in place of HMS. Prostaglandins, long chain unsaturated fatty acid have been closed up as important mediators in cell function. In platelets, $\mathrm{PGE}_{1}$ acts as an activator of adenylate cyclase in the platelet membrane, bringing about increase of cyclic AMP production. $\mathrm{PGE}_{1}$ of $10^{-4}$ to $10^{-6} \mathrm{M}$ concentration inhibited platelet aggregation induced by ADP, pinephrine, collagen, by inhibiting release reaction. Addition of $\mathrm{PGE}_{1}$ of these concentrations did not alter the basal production of $\mathrm{CO}_{2}$ via $\mathrm{CAC}$ but rather increased lactate production from anaerobic glycolysis. $\mathrm{PGE}_{1}$ of $10^{-8} \mathrm{M}$ concentration did not act as an inhibitor any more. $\mathrm{PGE}_{2}$ which was produced from arachidonic acid, behaves differently in platelets. Recently, arachidonic acid and their intermediates; cyclic endoperoxides and $\mathrm{PGE}_{2}$ are proved as active substance to sensitize platelets aggregability. The effect of $\mathrm{PGE}_{2}$ on platelet function and metabolism were studied. To evaluate the risk of thrombosis in increased number of platelets in CML, MMM and primary thrombocythemia, metabolic and kinetic study were done as well as adhesion and aggregation. Those platelets which responded abnormally to epinephrine, sometimes to collagen, but reacted to ADP, ristocetin normally, were incubated with $\mathrm{PGE}_{2}$, resulting in partial or total improvement of abnormal aggregation pattern by epinephrine to the normal. In CML, basal production of $1.27 \mathrm{n}$ moles increased to $1.88(\times 1.5)$ by epinephrine, to $2.43(\times 1.9)$ by epinephrine plus $\mathrm{PGE}_{2}$. In MMM, basal level of 2.51 n moles did not increase by epinephrine alone but some increase by preincubation with $\mathrm{PGE}_{2}$ to $3.18 \mathrm{n}$ moles $(\times 1.3)$. These findings suggested abnormality in plate. lets not only quantitative; marked in. crease in number, but also qualitative. These abnormalities, however, might be effective for self control mechanism to reduce high risk of aggregability due to increased number. Kinetic study on those cases with this abnormality, was done on a case of MMM who showed short survival ( $\mathrm{T} 1 / 2=1.75 \mathrm{~d}$.) with increased turnover and decreased recovery. A case of CML and a case of primary thrombocythemia exhibited normal life span.

Similar abnormalities in aggregation were seen in cases of long term administration of anti-platelets agents; dipyridamole and aspirin, after operation of artificial valve replacement. To predict an episodes of thrombosis as early as possible is most important problems in their aftercare. On 11 cases of those patients, short survivals ( $\mathrm{T} 1 / 2) ; 3.64 \pm$ 0.70 days in mirtal valve replacement (MVR), and $3.30 \pm 0.67$ days in aortic valve replacement (AVR): with decreased recovery more pronuonced in AVR than in MVR, were proved suggesting increased sequestration and consumption. Turnover of platelets also increased slightly in MVR $\left(4.65 \pm 2.30 \times 10^{4} / \mathrm{cmm} /\right.$ day $)$ 
but marked in AVR $\left(7.55 \pm 2.73 \times 10^{4}\right)$, suggesting compensated active thrombopoiesis.

\section{Conclusion}

Among those parameters, short survival less than 3 days, was helpful evidence to predict thrombotic episodes in two cases, indicating increased consumption. It was also suggested that intrinsic change due to drugs could be involved and prompted us metabolic study on those platelets (Thromb Res Suppl
2, 1976 in press). Thus in conclusion, we presented in this study, essential process of platelet plug formation; release reaction and aggregation are energy dependent. Therapeutic trials and analysis of self control mechanism of this process have been effective and promissing for prophylaxis. Aspirin and other antipyretics have been proved potent inhibitors of $\mathrm{PGE}_{2}$ biosynthesis in platelets, and establishement of ioslation and determination of these fatty acid or metabolites are in progress. 